\title{
Development and Evaluation of an Educational Computer Game for a Certification Examination
}

\author{
Gwo-Haur Hwang \\ Chen-Yu Lee \\ Wei-Fang Tseng \\ Ling Tung University
}

\begin{abstract}
Professional certification has become one of the important criteria for people to apply job positions in industries. Recently, computer-based learning has been widely adopted for helping students prepare for various professional certifications. However, most of the computerbased learning materials are not attractive to students, such that their learning performance is significantly affected owing to the lack of learning interests. Researchers have presented that joyful game-based learning can increase learner's motive and pleasure. Therefore, this study builds a certification tutorial system containing two different modules that are the traditional e-version and the joyful game-based version. Moreover, an experiment has been conducted to explore the participants' engagement and learning effectiveness of the proposed approach. Some terms are explained that the "prior knowledge" is represented by the "pre-test," and the "level of effort" (equivalent to the "engagement") is represented by the "experience" which is the cumulative number of correct answers. The result indicates that the traditional e-version may be slightly helpful for those participants with higher/middle prior knowledge and level of effort, and the joyful game-based version may be more helpful for those participants with lower prior knowledge and level of effort.
\end{abstract}

Keywords: professional certification, computer-based test, joyful game-based learning, learning effectiveness

\section{Introduction}

Shanker (1996) considered the certification mechanism a guarantee of basic profession for the owners in their field. Luo (2006) considered that the professional certificate is one of key factors to break up the diploma disease. Many western enterprises hire employees not only by the diploma, but also by the professional certificates (retrieved from http://www.merit-times. com.tw/NewsPage.aspx? Unid=30952).
Peng (2003) further pointed out that the IT certificate has become an important reference of employment.

The advancement of computer technology has emerged a lot of studies related to computer-based learning. Based on a review of previous studies, Bugbee and Alan (1996) pointed out that the advantages of computerbased tests include time reduction, raising security, and real-time reporting of results. The computer-based test is more accurate 
than the paper-based test. Announced by the Examination Yuan in Taiwan (2003), a computer-aided approach was adopted by the TOEFL test as well as the future planning of national examinations (retrieved from http:// www.saec.edu.tw/journal/163c.htm).

Along with the maturity of Internet technology, McCormack and Jones (1997) mentioned that the Web-based test improves the process and the approach of assessment. Because the Web-based test possesses the feature of time reduction, immediate feedback, resource saving, record archiving, and more convenience, assessment is made easier to complete.

Prensky (2001) proposed that learning integrated with the characteristics of game was able to increase the user's curiosity, intrinsic motive, and therefore learning effectiveness. The result of Hsiao and $\mathrm{Wu}$ (2003) indicated that the creativity of elementary school students was promoted more than the traditional approaches by a teaching model integrated with the characteristics of online game.

According to the above literature, the professional certificate has already become one of the critical employment criteria, and the trend is toward using the computer-based test instead of the paper-based test. Due to the rapid development of Internet and computer games, a lot of research related to the Webbased learning and game-based learning has been performed. Therefore, this study has developed an educational computer game for the certification examination of e-commerce by investigating its learning effectiveness as compared with the traditional approach.

\section{Literature Review}

\subsection{Certification Examination Tutorial}

Devedzic (2003) indicated that, owing to the advancement and popularity of information technologies, Web-based learning and the Web- based testing have become important issues in education. He further indicated that technologyenhanced learning could be helpful to students if appropriate feedback was provided. Chen (2004) attempted to combine assessment with the Internet technology in his study. The results showed that there was a positive impact on students' learning motive in such a Web-based learning environment; that is, an appropriate network interface design was able to raise learning performance, learning interests, and learning outcomes of students. Yeh (2006) integrated the tutor platform of a financial certification examination with interactive computer-based learning and testing to improve a shortage of interactivity and immediate feedback when using a paper-based approach. Lee (2006) digitized the content of certification questions and adopted an on-line test instead of a paper-based test. The results indicated that the learning effectiveness of students using the on-line test was obviously better than those of students using the paper-based test. That was because the on-line test, by means of the highspeed processing capabilities of computers, enables students to review in time and raise their practice efficiency.

\subsection{Game-Based Learning}

Hsiao and $\mathrm{Wu}$ (2003) considered that the online games possess the characteristics of curiosity, imagination, adventure, challenge, competition, and in-time synchronization to attract players' participation. These characteristics provide learners with a considerable motive to adopt the online gamebased learning. Wang, Wang, Wang, and Huang (2004) developed the game assessment module of the Web-based assessment and test analysis system (GAM-WATA), and in this module it provided students with an online help function named ASK-HINT. This function increased the interest of assessment and made the participants more willing to actively participate. Chuang (2004) assumed that the students had a good 
impression of digital game-based learning before, and this made digital game-based learning more acceptable than others. Tsai, $\mathrm{Yu}$, and Hsiao (2008) found learning tasks and interactivity in the digital game-based learning produced a key influence on the learning behavior and learning effectiveness of its users, and it also demonstrated the value of digital game-based learning in specific learning field.

Chang and Chen (2009) found that participants from Taiwan experienced increases in both content learning and engagement when using video game-based learning as opposed to text-based computer aided instruction. Dedeaux and Hartsell (2011) mentioned that the more similar to successful commercial video games the educational computer games are designed, the more engaging and effective the game will be for the user. In addition, greater engagement can lead to greater achievement. The results of their study also implied that the most effective games are difficult, even frustrating, and involve a certain degree of action, fast-paced decision making, and hand-eye coordination.

To sum up the above, this study builds an educational computer game for the certification examination of e-commerce to explore the participants' engagements and the effectiveness of game-based learning. The system is designed to contain two different modules which are the traditional e-version and the joyful game-based version to analyze the difference of learning effectiveness for participants under these two versions. According to the features of the most effective games proposed by Hsiao and $\mathrm{Wu}(2003)$ as well as Dedeaux and Hartsell (2011), some elements are added in this computer game. For example, the multimedia including animation and sound effect increase the interest of the participants, the life balls increase the challenge of the participants, the score ranking increases the competition among the participants, and the experience demonstrates the participant's engagement. These game elements are intended to make the educational computer game more similar to successful commercial video games to increase the participants' engagement.

\section{System Introduction}

\subsection{System Architecture}

The system development tools include Adobe FLASH for the front-end interface design, PHP for Web page interconnection and MySql for the database management system. The hardware architecture is illustrated in Figure 1. This system provides teachers management functions of student data, learning profile, and game chapter. A gamebased learning version is provided to students with relative functions including chapter selection, learning profile, and individual score inquiry. There are four database files which include chapter question, learning profile, chapter score, and student data. The software architecture is illustrated in Figure 2.

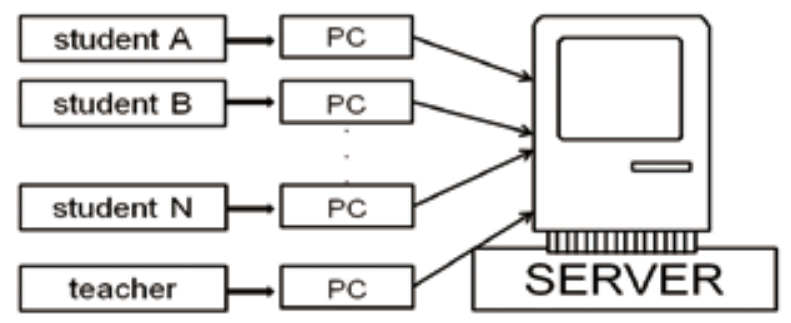

Figure.1 Hardware architecture. 


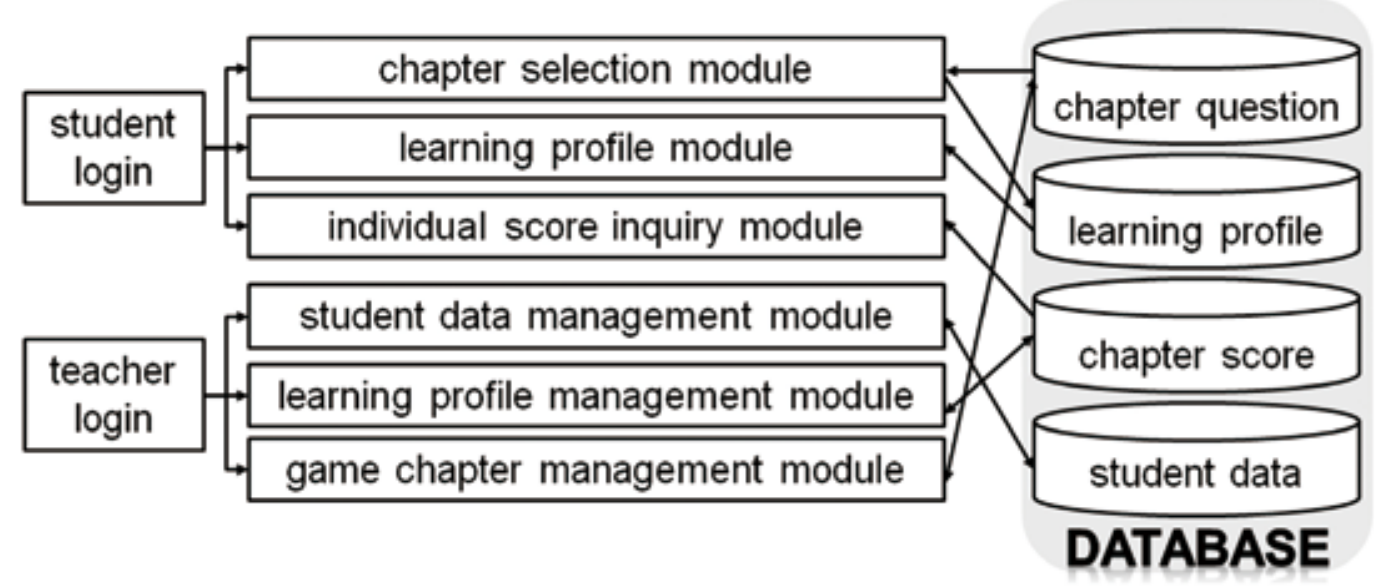

Figure.2 Software architecture.

\subsection{Educational Computer Game}

The system named "qualified road" with $\mathrm{QR}$ as its acronym is proposed to serve as the tutor of an e-commerce certification examination, and it is composed of a traditional e-version TQR and a game-based joyful version JQR, which is an educational computer game. When the participants login into the $\mathrm{QR}$, they will be distinguished into either users of TQR or JQR, and then be directed to the corresponding version.
Functions in both versions include: (a) "individual score inquiry" can be used to look up the average score in each chapter, (b) "answered question record" provides records of answered questions with wrong answer and abandoned questions in each chapter, and (c) "chapter selection" contains nine chapters corresponding to eight categories of questions in the textbook and a comprehensive test. There are two additive functions only in JQR: the "chapter score ranking" and the "experience ranking." The TQR menu is illustrated in Figure 3 , and the JQR menu is illustrated in Figure 4.

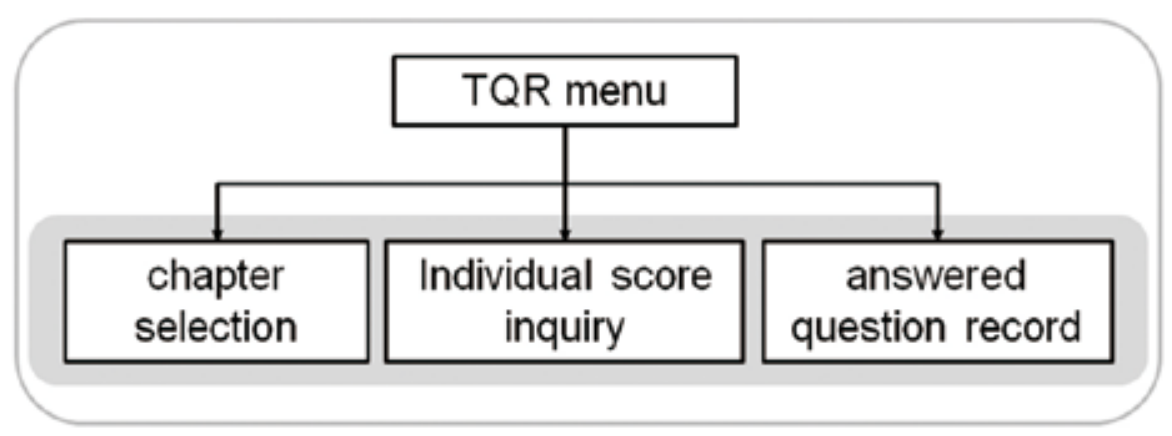

Figure 3. The TQR menu. 


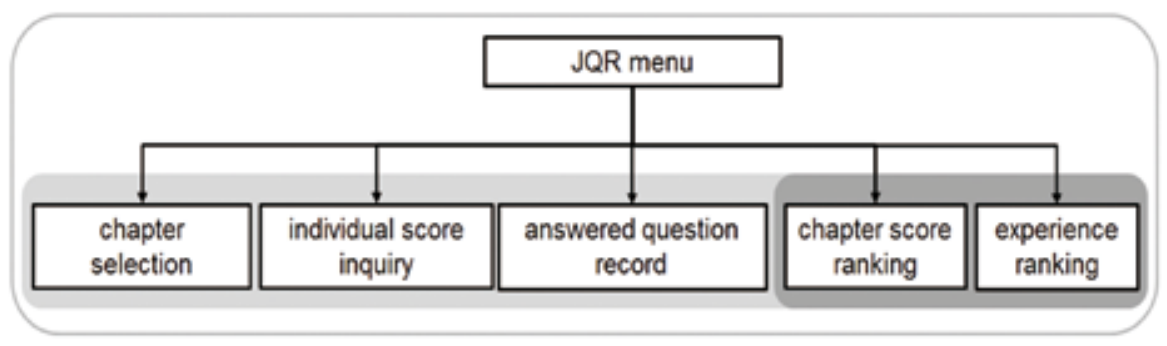

Figure 4. The JQR menu.

Some test rules for both versions include: (a) there are total 50 questions in each chapter, (b) the test time is 40 minutes, (c) there are two types of questions in each chapter, the single-choice and the multiple-choice questions, (d) two points are obtained for correct answer, and one point is lost if answer is wrong, (e) when one question is completed, it will proceed directly to the next, and (f) the obtained points must be equal to or greater than 70 to pass the examination. In addition, three elements are added only in the JQR including "life ball", "experience", and "abandon answering".
The life ball with an initial value of 30 represents the upper bound of lost points to conquer the game. The experience is cumulative without limits and represents the engagement or the level of effort. The mechanism is getting one point of experience when giving the correct answer; losing three life balls when getting wrong answer; getting a loss of two life balls when abandoning to answer the current question and proceed directly to the next. The comparison of test rules for TQR and JQR is listed in Table 1.

Table 1. The Comparison of Test Rules for TQR and JQR

\begin{tabular}{lll}
\hline & TQR & JQR \\
\hline Time limits & 40 minutes & 40 minutes \\
Total questions & 50 questions & 50 questions \\
Initial state & - & 30 life balls \\
Correct answer & increase two score points & $\begin{array}{l}\text { increase two score points and } \\
\text { get one experience point } \\
\text { decrease one score point and } \\
\text { lose three life balls } \\
\text { lose two life balls }\end{array}$ \\
Abandon answering & decrease one score point & \\
\hline
\end{tabular}

In TQR, the score is calculated when all of the questions are completely answered or the time is exhausted. In JQR, if the score is equal to or greater than 70 when exhausting the time, the game is conquered and the score is recorded. If the score is smaller than 70 when exhausting the time or the life balls have ran out within time limits, the user has failed the game without a recording of the score. The comparison of features for TQR and JQR is listed in Table 2. 
Table 2. The Comparison of Features for TQR and JQR

\begin{tabular}{lcc}
\hline & TQR & JQR \\
\hline Multimedia, animation and sound effect (the interest) & $\mathrm{X}$ & $\mathrm{V}$ \\
$\begin{array}{l}\text { Display the current score in the test } \\
\begin{array}{l}\text { The loss of points make an influence on the chance to proceed (the } \\
\text { chanllenge) }\end{array}\end{array}$ & $\mathrm{V}$ & $\mathrm{V}$ \\
$\begin{array}{l}\text { According to the mechanism, the test is forced to terminate without } \\
\text { recording the score }\end{array}$ & $\mathrm{X}$ & $\mathrm{V}$ \\
$\begin{array}{l}\text { The answered question record } \\
\text { The individual score inquiry }\end{array}$ & $\mathrm{V}$ & $\mathrm{V}$ \\
$\begin{array}{l}\text { The chapter score ranking (the chanllenge) } \\
\text { The experience ranking (the chanllenge) }\end{array}$ & $\mathrm{X}$ & $\mathrm{V}$ \\
\end{tabular}

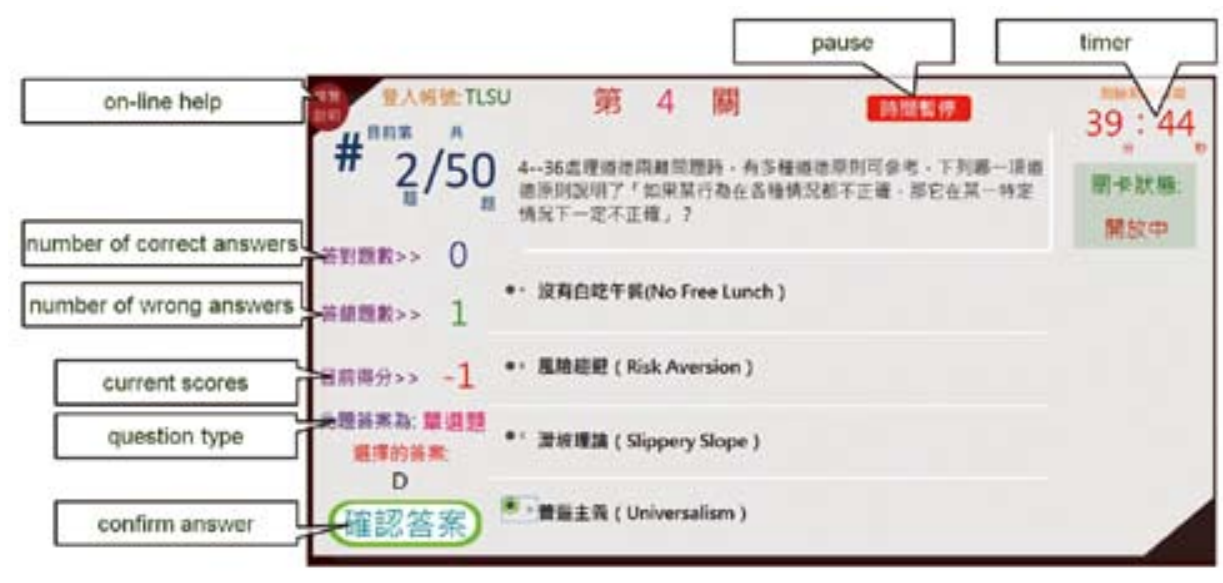

Figure 5. The TQR user interface

The user interfaces of TQR and JQR are illustrated in Figure 5 and Figure 6, respectively. Some elements of the user interface are marked up with the caption boxes. 


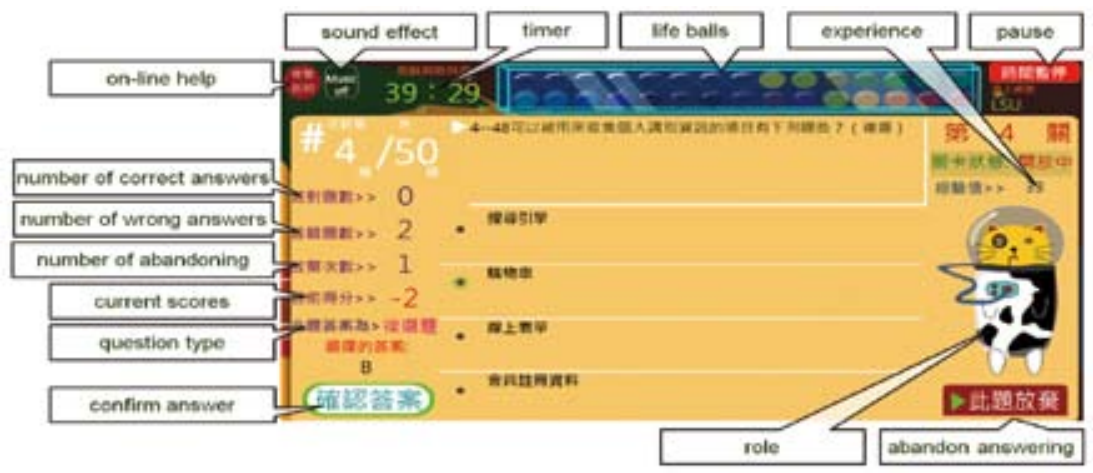

Figure 6. The JQR user interface

\section{Experiment Design}

\subsection{Experiment Planning}

The experiments took place between April, 2012 and June, 2012. The participants in the experiments included two classes of sophomores from a university of science and technology in the middle of Taiwan. These two classes were divided into a TQR group with 51 participants and a JQR group with 56 participants (totally 107 participants), respectively. Some conditions were controlled that the teaching content and the teacher were identical to all participants. The scores of pretest and post-test were used to analyze the difference of learning effectiveness between TQR group and JQR group.

\subsection{Experiment Implementation}

Before launching the experiment, a pre-test was implemented and the operation of the tutorial was explained. The purpose of a pre-test was to investigate prior knowledge of participants. In the experiment, the textbook and the teacher were identical to all participants, and the corresponding test was accessible for all participants after one chapter had been lectured upon completely. All participants were supposed to complete the test of the chapter within the accessible period of time. The length of the experiment was planned to last for nine weeks. Then, the post-test would be implemented to assess the learning effectiveness of all participants, and to analyze the difference of the learning effectiveness between the TQR group and the JQR group. The Experiment implementation flowchart is illustrated in Figure 7.

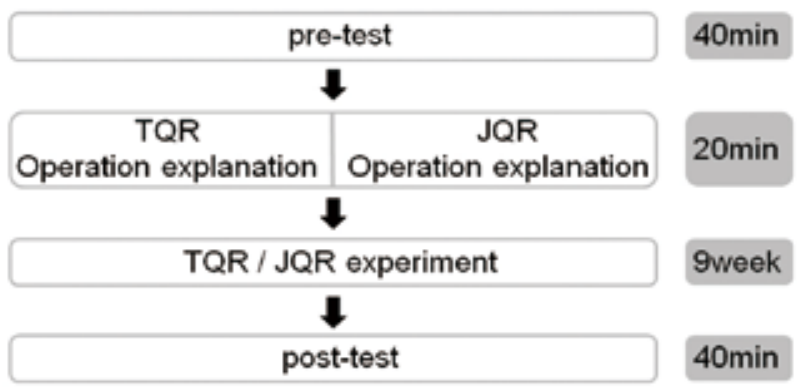

Figure 7. Experiment implementation flowchart. 


\section{Experimental Analysis}

In this study, the objects to be analyzed were those participants who have taken the pre-test, used the system, and taken the posttest. Thus, 23 participants for the JQR group and 35 participants for the TQR group were finally filtered as objects. The differences of learning effectiveness between the JQR group and the TQR group were explored, and the advanced analyses of paired JQR-TQR groups classified by the prior knowledge and the level of effort were also prepared as below.

The "prior knowledge" was represented by the "pre-test" and abbreviated as PK. In order to make further analyses, the prior knowledge was used to classify the JQR group and the TQR group, and the classification process included the following steps. First, the PK for JQR group and TQR group were sorted into descending order respectively. Then, one-third in the front was adopted as the group of higher $\mathrm{PK}$, one-third in the middle was adopted as the group of middle PK, and one-third in the rear was adopted as the group of lower PK. Therefore, the process generated three pairs as follows: "JQR(H-PK)" vs. "TQR(HPK)", “JQR(M-PK)” vs. “TQR(M-PK)”, and "TQR(L-PK)" vs. "TQR(L-PK)." The classification and paired groups by the prior knowledge (PK) are illustrated in Figure 8.

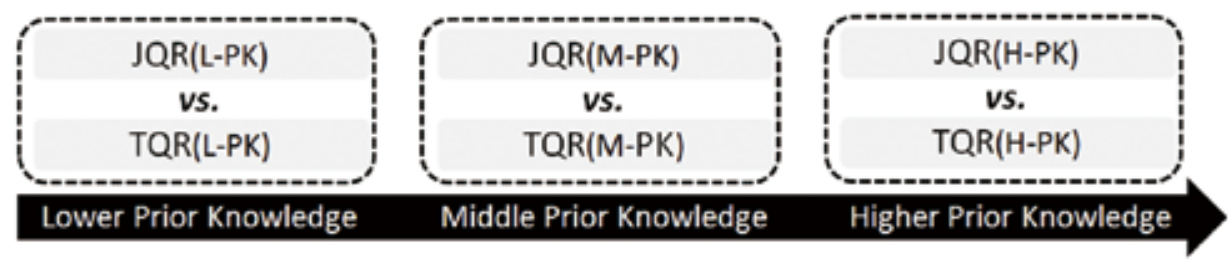

Figure 8 . The classification and paired groups by the prior knowledge.

The "level of effort" or the "engagement" was represented by the "experience" and abbreviated as LOE. In order to make further analyses, the level of effort was used to classify the JQR group and the TQR group, and the classification process included the following steps. First, the LOE for JQR group and TQR group were sorted into descending order respectively. Then, one-third in the front was adopted as the group of higher LOE, onethird in the middle was adopted as the group of middle LOE, and one-third in the rear was adopted as the group of lower LOE. Therefore, the process generated three pairs as follows: "JQR(H-LOE)" vs. "TQR(H-LOE)", “JQR(MLOE)" vs. "TQR(M-LOE)", and “JQR(LLOE)" vs. "TQR(L-LOE)." The groups with the identical nature for JQR and TQR were compared in pairs. The classification and paired groups by the level of effort (LOE) are illustrated in Figure 9.

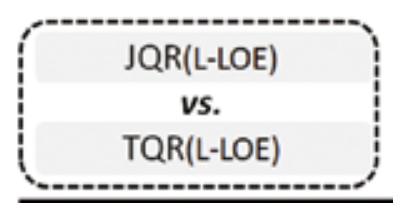

Lower Level of Effort

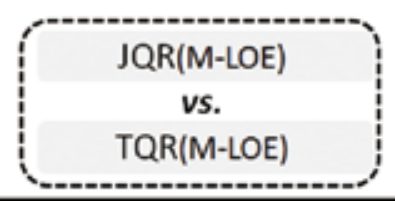

Middle Level of Effort

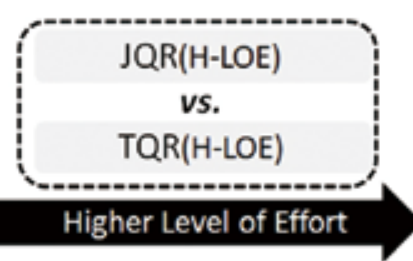

Figure 9. The classification and paired groups by the level of effort. 


\subsection{Exploration Question}

According to the classification mentioned above, three questions explored in the study were: (1) Are there differences of learning effectiveness between the JQR group and TQR group? (2) Are there differences of learning effectiveness between paired JQRTQR groups with higher prior knowledge, middle prior knowledge, and lower prior knowledge respectively? and (3) Are there differences of learning effectiveness between paired JQR-TQR groups with higher level of effort, middle level of effort, and lower level of effort respectively?

\subsection{Question Analysis}

As to the exploration questions mentioned above, the corresponding analyses were made and discussed in the following subordinate sections.

\subsubsection{Difference of Learning Effectiveness for JQR Group and TQR Group}

The value of progress was calculated by subtracting the pre-test score from the posttest score. The pre-test, post-test, and progress for the JQR group and the TQR group were estimated by the $t$-test and illustrated in Table 3 .

Table 3. The Estimation of Pre-test, Post-test and Progress for JQR Group \& TQR Group

\begin{tabular}{lllllll}
\hline \multirow{2}{*}{ Pre-test } & Group & N & Mean & S.D. & $p$ & $t$ \\
& JQR & 23 & 33.30 & 7.022 & .474 & -1.281 \\
\multirow{3}{*}{ Post-test } & TQR & 35 & 36.03 & 8.456 & & \\
& JQR & 23 & 76.87 & 17.426 & .565 & .456 \\
& TQR & 35 & 74.46 & 21.041 & & \multirow{2}{*}{.027} \\
& JQR & 23 & 43.57 & 18.278 & .850 & \\
& TQR & 35 & 38.43 & 18.851 & & \\
\hline
\end{tabular}

Table 4. The Estimation of Pre-test, Post-test and Progress for JQR(H-PK) \& TQR(H-PK)

\begin{tabular}{lcccccc}
\hline \multirow{2}{*}{ Pre-test } & Group & $\mathrm{N}$ & Mean & S.D. & $p$ & $t$ \\
& JQR & 6 & 42.17 & 3.312 & .380 & -1.111 \\
& TQR & 11 & 45.00 & 5.692 & & \\
\multirow{5}{*}{ Post-test } & JQR & 6 & 80.00 & 13.100 & .201 & -.547 \\
& TQR & 11 & 82.73 & 7.682 & & .018 \\
& JQR & 6 & 37.83 & 14.878 & .285 & \\
& TQR & 11 & 37.73 & 9.045 & & \\
\hline
\end{tabular}


The result indicated that the difference of the pre-test score between the JQR group and the TQR group was not significant, and it meant that there was an equivalent capability for two groups before the experiment. The results of the post-test score and the progress for two groups were also not significant, and it meant that there was no significant difference of learning effectiveness for two groups after the experiment. However, if observing the mean of the post-test score and the progress, the JQR group was correspondingly 2.41 and 5.14 higher than the TQR group. This implied that the learning effectiveness of the JQR group was slightly better than the learning effectiveness of the TQR group.

\subsubsection{Difference of Learning Effectiveness between Paired JQR-TQR Groups by Different Prior Knowledge}

In this section, the differences of learning effectiveness between the paired JQR-TQR groups by the higher/middle/lower prior knowledge are discussed.

The pre-test, post-test, and progress of the paired groups which were composed of JQR(H-PK) and TQR(H-PK) were estimated by the $t$-test and illustrated in Table 4 .

Table 5. The Estimation of Pre-test, Post-test and Progress for JQR(M-PK) \& TQR(M-PK)

\begin{tabular}{llllccc}
\hline \multirow{2}{*}{ Pre-test } & Group & $\mathrm{N}$ & Mean & S.D. & $p$ & $t$ \\
& JQR & 10 & 33.60 & 2.366 & .120 & -3.898 \\
& TQR & 11 & 37.18 & 1.834 & & \\
\multirow{5}{*}{ Post-test } & JQR & 10 & 73.30 & 20.678 & .178 & -1.363 \\
& TQR & 11 & 82.27 & 6.828 & & \\
& JQR & 10 & 39.70 & 19.794 & .225 & -.842 \\
& TQR & 11 & 45.09 & 7.449 & & \\
\hline
\end{tabular}

The result indicated that the differences of the pre-test, post-test, and progress were not significant, and it meant that an equivalent capability existed before the experiment and no significant difference of learning effectiveness occurred after the experiment for two groups.

The pre-test, post-test and progress of the paired groups which were composed of JQR(M-PK) and TQR(M-PK) were estimated by the $t$-test and illustrated in Table 5 .

The result indicated that the differences of the pre-test, post-test, and progress were not significant, and it meant that an equivalent capability existed before the experiment and no significant difference of learning effectiveness occurred after the experiment for two groups. However, if observing the mean of the post-test score and the progress, the TQR (M-PK) group was correspondingly 8.97 and 5.39 higher than the JQR (M-PK) group. This implied that the learning effectiveness of the TQR(M-PK) group was slightly better than the learning effectiveness of the JQR(M-PK) group.

The pre-test, post-test, and progress of the paired groups which were composed of JQR(L-PK) and TQR(L-PK) were estimated by the t-test and illustrated in Table 6 . 
Table 6. The Estimation of Pre-test, Post-test and Progress for JQR(L-PK) \& TQR(L-PK)

\begin{tabular}{lcccccc}
\hline \multirow{2}{*}{ Pre-test } & Group & $\mathrm{N}$ & Mean & S.D. & $p$ & $t$ \\
& JQR & 7 & 25.29 & 3.094 & .286 & -1.184 \\
& TQR & 13 & 27.46 & 4.274 & & \\
\multirow{3}{*}{ Post-test } & JQR & 7 & 79.29 & 17.066 & .090 & 1.785 \\
& TQR & 13 & 60.85 & 29.103 & & \multirow{2}{*}{.045} \\
& JQR & 7 & 54.00 & 16.371 & .098 & \\
& TQR & 13 & 33.38 & 28.701 & & \\
\hline
\end{tabular}

The result indicated that the differences of the pre-test, post-test, and progress were not significant, and it meant that an equivalent capability existed before the experiment and no significant difference of learning effectiveness took place after the experiment for two groups. However, if observing the mean of the post-test score and the progress, the JQR(L-PK) group was correspondingly 18.44 and 20.62 higher than the TQR(L-PK) group, and it implied that the learning effectiveness of the JQR(L-PK) group was seemingly better than the learning effectiveness of the TQR(L-PK) group.

\subsubsection{Difference of Learning Effectiveness between Paired JQR-TQR Groups by Different Level of Effort}

In this section, the differences of learning effectiveness between the paired JQR-TQR groups by the higher/middle/lower level of effort are discussed as follows.

The pre-test, post-test, and progress of the paired groups which were composed of JQR(H-LOE) and TQR(H-LOE) were estimated by the $t$-test and illustrated in Table 7 .

Table 7. The Estimation of Pre-test, Post-test And Progress for JQR(H-LOE) \& TQR(H-LOE)

\begin{tabular}{|c|c|c|c|c|c|c|}
\hline & Group & $\mathrm{N}$ & Mean & S.D. & $p$ & $t$ \\
\hline \multirow[t]{2}{*}{ Pre-test } & JQR & 8 & 35.13 & 5.357 & .198 & -.713 \\
\hline & TQR & 13 & 37.69 & 9.223 & & \\
\hline \multirow[t]{2}{*}{ Post-test } & JQR & 8 & 78.63 & 12.603 & .293 & -.851 \\
\hline & TQR & 13 & 82.46 & 8.171 & & \\
\hline \multirow[t]{2}{*}{ Progress } & JQR & 8 & 43.50 & 16.353 & .718 & -.201 \\
\hline & TQR & 13 & 44.77 & 12.531 & & \\
\hline
\end{tabular}


The result indicated that the differences of the pre-test, post-test, and progress were not significant, and it meant that an equivalent capability existed before the experiment and no significant difference of learning effectiveness arose after the experiment for the paired groups. However, if observing the mean of the post-test score and the progress, the TQR(H-LOE) group was correspondingly 3.83 and 1.27 higher than the JQR(H-LOE) group. This implied that the learning effectiveness of the TQR $(\mathrm{H}-\mathrm{LOE})$ group was slightly better than the learning effectiveness of the JQR(H-LOE) group.

The pre-test, post-test, and progress of the paired groups which were composed of JQR(MLOE) and TQR(M-LOE) were estimated by the $t$-test and illustrated in Table 8 .

Table 8. The Estimation of Pre-test, Post-test and Progress for JQR(M-LOE) \& TQR(M-LOE)

\begin{tabular}{lcccccc}
\hline & Group & $\mathrm{N}$ & Mean & S.D. & $p$ & $t$ \\
\hline \multirow{2}{*}{ Pre-test } & JQR & 7 & 34.00 & 8.660 & .312 & -.906 \\
& TQR & 12 & 37.25 & 6.851 & & \\
\multirow{5}{*}{ Post-test } & JQR & 7 & 73.00 & 14.422 & .967 & -.733 \\
& TQR & 12 & 78.67 & 17.169 & & \\
& JQR & 7 & 39.00 & 9.764 & .299 & -.347 \\
& TQR & 12 & 41.42 & 16.725 & & \\
\hline
\end{tabular}

The result indicated that the differences of the pre-test, post-test, and progress were not significant, and it meant that an equivalent capability existed before the experiment and no significant difference of learning effectiveness arose after the experiment for the paired groups. However, if observing the mean of the posttest score and the progress, the TQR(M-LOE) group was correspondingly 5.67 and 2.42 higher than the JQR(M-LOE) group. This implied that the learning effectiveness of the TQR(MLOE) group was slightly better than the learning effectiveness of the JQR(M-LOE) group.

The pre-test, post-test, and progress of the paired groups which were composed of JQR(LLOE) and TQR(L-LOE) were estimated by the $t$-test and illustrated in Table 9.

Table 9. The Estimation of Pre-test, Post-test and Progress for JQR(L-LOE) and TQR(L-LOE)

\begin{tabular}{lcccccc}
\hline \multirow{2}{*}{ Pre-test } & Group & $\mathrm{N}$ & Mean & S.D. & $p$ & $t$ \\
& JQR & 8 & 30.88 & 7.140 & .234 & -.394 \\
\multirow{3}{*}{ Post-test } & TQR & 10 & 32.40 & 8.872 & & \\
& JQR & 8 & 78.50 & 24.407 & .284 & 1.517 \\
& TQR & 10 & 59.00 & 29.010 & & \\
& JQR & 8 & 47.63 & 25.712 & .795 & \\
& TQR & 10 & 26.60 & 23.787 & & \\
\hline
\end{tabular}


The result indicated that the differences of the pre-test, post-test, and progress were not significant, and it meant that an equivalent capability existed before the experiment and no significant difference of learning effectiveness took place after the experiment for the paired groups. However, if observing the mean of the post-test score and the progress, the JQR(LLOE) group was correspondingly 19.5 and 21.03 higher than the TQR(L-LOE) group. This implied that the learning effectiveness of the JQR (L-LOE) group was seemingly better than the learning effectiveness of the TQR(L-LOE) group. It was also observed that although the differences of the mean of the post-test score and the progress for the JQR (L-LOE) group from the TQR (L-LOE) group was large enough, the divergence represented by the standard deviation (S.D.) may be too large to lead to no significance.

\section{Conclusion}

According to the analysis of the experiment, for all cases there were no significant differences of the learning effectiveness between the JQR group and TQR group or between the paired groups classified by the prior knowledge (PK) and the level of effort (LOE).

However, some findings were observed in the analysis of the experiment in spite of no significance. After comparing the mean of the post-test score and the progress respectively, the result implied that the learning effectiveness of the JQR group was slightly better than the learning effectiveness of the TQR group.

According to the classification by the prior knowledge, after comparing the mean of the post-test score, the result indicated that the TQR(H-PK) group was slightly higher than the JQR(H-PK) group, and the TQR(M-PK) group was also higher than the JQR(M-PK) group. It implied that the traditional e-version enables slightly higher score of the post-test for those participants with higher and middle prior knowledge. If comparing the mean of the progress, the JQR(H-PK) group was a little higher than the TQR(H-PK) group, but the TQR(M-PK) group was slightly higher than the JQR(M-PK) group.

According to the classification by the level of effort (equivalent to the engagement), after comparing the mean of the post-test score and the progress respectively, the result indicated that the TQR(H-LOE) group was slightly higher than the JQR(H-LOE) group; the TQR(M-LOE) group was also slightly higher than the JQR(M-LOE) group; and the JQR(L-LOE) group was seemingly higher than the TQR(L-LOE) group. It implied that the traditional e-version may enable slightly higher score of the post-test and slightly higher progress for those participants with higher and middle level of effort, but the joyful game-based version may be better for those participants with lower level of effort.

Therefore, according to the comprehensive analyses, the traditional e-version may be slightly helpful for those participants with higher/middle prior knowledge and level of effort, and the joyful game-based version may be more helpful for those participants with lower prior knowledge and level of effort.

\section{References}

Bugbee, J., \& Alan, C. (1996). The equivalence of paper-and-pencil and computer-based testing. Journal of Research on Computing in Education, 28(3), 282-289.

Chang, T., \& Chen, W. (2009). Effects of computer-based video games on children: An experimental study. Educational Technology \& Society, 12(2), 1-10. 
Chen, G. Y. (2004). Effects of pen-based and web-based tests on english testing performances of junior high school students (Master's thesis). Retrieved from http://ndltd.ncl.edu. tw/cgi-bin/gs32/gsweb.cgi/ccd=g5Er4x/ webmge? mode $=$ basic

Chuang, K. H. (2004). The study of application on web based game for learning of transformations of matter (Master's thesis). Retrieved from http:// ndltd.ncl.edu.tw/ cgi-bin/gs32/gsweb.cgi/ ccd $=$ g5 Er4x/webmge?mode=basic

Dedeaux, T. \& Hartsell, T. (2011). Educational computer games and Spanish content learning. Journal of Educational Technology Development and Exchange, 4(1), 55-70.

Devedzic, V. B. (2003). Key issues in nextgeneration web-based education. IEEE Trans-actions On Systems, Man, And Cybernetics-PART C: Applications and Reviews, 33(3), 339-349.

Hsiao, H. S., \& Wu, C. H. (2003). Building a creativity teaching strategy into the instructional of web-based games. Living Technology Education, 36(2), 38-52.

Lee, Y. S. (2006). The effectiveness of online test verses pen-based test-exemplified by test of academic proficiency in computer software application (Master's thesis). Retrieved from http://ndltd.ncl.edu.tw/cgibin/gs32/gsweb.cgi/ccd=g5Er4x/webmge? mode $=$ basic

McCormack, D., \& Jones, D. (1997). Building a Web-based Education System. New York, Wiley.

Peng, H. H., (2003). Study of promoting skill management and certificate system in knowledge-based service organization Case of industrial technology intelligence services \& promotion project (Master's thesis). Retrieved from http://ndltd. ncl.edu.tw/ cgi-bin/gs32/gsweb.cgi/ $\mathrm{ccd}=\mathrm{g} 5 \mathrm{Er} 4 \mathrm{x} /$ webmge$?$ mode $=$ basic
Prensky, M. (2001). Digital Game-based Learning. New York, McGraw-Hill.

Shanker, A. (1996). Quality assurance. PhiDeltaKappan, 78(3), 220-225.

Tsai, F. H., Yu, K. C., \& Hsiao, H. S. (2008). Discovering the value of digital gamebased learning from the new perspective of transfer. Curriculum \& Instruction Quarterly, 11(4), 237-278.

Wang, T. H., Wang, K. H., Wang, W. L., \& Huang, S. C. (2004). Assessing effectiveness of formative assessment models on students' learning achievement in e-learning environment. Living Technology Education, 4(2), 469-490.

Yeh, C. H. (2006). Constructing a computeraided system for financial certification examination - Taking investments for example (Master's thesis). Retrieved from http://ndltd.ncl.edu. tw/cgi-bin/gs32/gsweb.cgi/ccd=g5Er4x/ webmge?mode=basic

\section{Acknowledgements}

This study is sponsored by the Science Council of Taiwan under the contract no. NSC99-2511-S-275-001-MY3.

\section{Contact the Authors}

Gwo-Haur Hwang

Ling Tung University

E-mail: ghhwang@mail.ltu.edu.tw

\section{Chen-Yu Lee}

Ling Tung University

E-mail: cylee@teamail.ltu.edu.tw

\section{Wei-Fang Tseng}

Ling Tung University

E-mail: helen10675@yahoo.com.tw 